Journal of Computer Science 8 (7): 1143-1149, 2012

ISSN 1549-3636

(C) 2012 Science Publications

\title{
Multipath Routing Protocol for Effective Local Route Recovery in Mobile Ad hoc Network
}

\author{
${ }^{1}$ D. Jagadeesan and ${ }^{2}$ S.K. Srivatsa \\ ${ }^{1}$ Department of Computer Science and Engineering, \\ Sri Chandrasekharendra Saraswathi Viswa Mahavidyalaya University, \\ Kanchipuram, Tamil Nadu, India \\ ${ }^{2}$ St. Joseph College of Engineering, Chennai
}

\begin{abstract}
Problem statement: In mobile ad hoc networks, frequent mobility during the transmission of data causes route failure which results in route rediscovery. In this, we propose multipath routing protocol for effective local route recovery in Mobile Ad hoc Networks (MANET). In this protocol, each source and destination pair establishes multiple paths in the single route discovery and they are cached in their route caches. Approach: The cached routes are sorted on the basis of their bandwidth availability. In case of route failure in the primary route, a recovery node which is an overhearing neighbor, detects it and establishes a local recovery path with maximum bandwidth from its route cache. Results: By simulation results, we show that the proposed approach improves network performance. Conclusion: The proposed route recovery management technique prevents the frequent collision and degradation in the network performance.
\end{abstract}

Keywords: Mobile Ad hoc Network (MANET), route recovery, route discovery, routing protocol, route failure, multiple paths, pair establishes, route cache, cached routes

\section{INTRODUCTION}

Mobile Ad hoc Network (MANET): A selfconfigured network of mobile nodes associated with wireless links in order to organize a random topology is termed as mobile ad hoc networks. The nodes travel in the random manner. (Seethalakshmi et al., 2011). Quick deployment, strength, flexibility and essential support for mobility are some of the merits of the ad hoc networks. As ad hoc network is economically beneficial, it is utilized in the military application, collective and distributed computing, emergency services, wireless mesh and sensor networks and even in hybrid networks.

The wireless transmission limit has undergone an extension with the help of the ad hoc networks on account of multi-hop packet forwarding strategy. This can support the situation during the pre-build infrastructure cannot cope up with the application. There is no existence of stationary infrastructure of the base station or switching centers in ad hoc network. The mobile nodes within radio limits interact directly through wireless routes and those that are distant depend on the other nodes to act as routers.
Routing in MANET:

Categories of Routing Protocols in MANET: The routing protocols in MANET are classified as follows:

- Table-Driven Routing Protocols

- On-demand Routing Protocols (Suganthi and Tamilarasi, 2012; Keming and Yang, 2010).

Issues related to MANET routing: The issues related to MANET routing are unpredictable of environment, unreliability of wireless medium, resource-constrained nodes and dynamic topology. These issues may result in faults such as transmission errors, node failures, link failures, route breakages, congested nodes or links (Khetwal and Sharma, 2012).

Route maintenance and recovery in MANET: Route discovery, route maintenance and traffic allocation are the three components includes in the multipath routing. The first two components establish of multiple routes between source and destination node. Moreover the multipath routing protocol tries finding disjoint nodes, disjoint link and non-disjoint routes. After the route is established, the mobile node starts forwarding the data

Corresponding Author: D. Jagadeesan, Department of Computer Science and Engineering,

Sri Chandrasekharendra Saraswathi Viswa Mahavidyalaya University, Kanchipuram,

Tamil Nadu, India 


\section{J. Computer Sci., 8 (7): 1143-1149, 2012}

packets to the destination. Usually during some situation, the route failure causes the forwarded packets to be lost. Other situations cause the packets to reach the destination with some delay. To tackle this problem, route maintenance technique is considered.

Route caching in MANET: The recently discovered routes should be cached for using it again when the similar route is demanded. The two types of route caching technique available for on-demand routing protocol are source route caching and intermediate route caching. The on-demand routing protocols such as AODV and DSR permits the intermediate node which has cached route to the destination respond to the source with the cached route. The route cache is necessary for granting forceful recovery in MANET. The merits of using route cache include the availability of the alternate route during link failure and controlling overhead which is necessary to repair the route.

Problem Identification: Traditional on-demand routing protocols produce a large amount of routing control traffic by blindly flooding the entire network with RREQ packets during route discovery. The routing overhead associated with the dissemination of routing control packets can be quite huge, especially when the network topology frequently changes (Aminu et al., 2009).

Multipath routing protocols cache multiple routes to a destination in a single route discovery. However, in the presence of mobility, multipath protocols incur additional packet drops and delay due to their dependency on potentially stale routes from caches Protocols using either limited broadcast or local error recovery have focused on reducing packet drops and not on utilizing the bandwidth efficiently during route recovery.

We propose to develop a hybrid routing protocol involving multipath discovery and local error-recovery. In this protocol, each source and destination pair establishes multiple paths in the single route discovery and they are cached in their route caches. The cached routes are sorted on the basis of their bandwidth availability.

Whenever a link or a route break is occurring, a local error-recovery is performed which in turn invokes the alternate route selection. An effective alternate route is selected from the route cache which is more consistent and having greater available bandwidth.

So our proposed hybrid routing technique has the following advantages:

- Reduces packet drops

- Reduces the recovery time

- Reduces overhead

- Utilizes bandwidth efficiently
Related works: Kang and Ko (2010) have proposed a location-based hybrid routing protocol to improve data packet delivery and to reduce control message overhead in mobile ad hoc networks. In mobile environments, where nodes move continuously at a high speed, it is generally difficult to maintain and restore route paths. Therefore, their study suggests a new flooding mechanism to control route paths. The essence of their proposed scheme is its effective tracking of the destination's location based on the beacon messages on the main route nodes.

Aminu et al. (2009) proposed a new probabilistic route discovery method for routing in MANETs, referred to as Probabilistic Counter-based Route discovery (PCBR), which combine the features of counter-based and gossip-based approaches. The performance of PCBR is evaluated using AODV as the base routing protocol, which traditionally uses the blind flooding. The effect of traffic load, mobility and topology size on the performance of the PCBR-AODV route discovery is not considered.

Dhirendra et al. (2010) have enhanced the performance of Split Multipath Routing protocols by using route update mechanism. Their proposal is useful in the route recovery process. In MANET for sending the data packets through alternate path takes more time in comparison with stale route that was broken. So, they repair the broken route through a route update mechanism process and reduce the delay through the new updated path. In their proposal they are considering the high gain antenna terminal that adjusts transmission range of each node and follow a new technique for route update mechanism. So, they provide a heuristic approach to reduce the delay metric and increase the performance of MANET.

Wu et al. (2009) have proposed a power saving routing protocol with power sieving technique in wireless ad hoc networks. They partitioned the network area into several square grids using Global Position System (GPS). The routing is performed in a grid-bygrid manner. One node is elected as the great leader in its grid with a power sieving mechanism without broadcasting election packets. The advantage of this technique is that it saves more power for data transmission and the network lifetime is also prolonged.

Juanwei et al. (2009) have proposed a new multipath protocol called the Multipath Routing Protocol for Networks Lifetime Maximization in AdHoc Networks (MRNLM) is proposed based on AOMDV. The protocol sets the energy threshold to optimize the forwarding mechanism. At the same time it builds an energy-cost function and uses the function as the criterion for multiple path selection. In transmission phase, they use a novelty method called "data transmission in multiple paths one by one" to balance the energy in multiple paths. 
Sung-Won et al. (2010) proposed a reliable and hybrid multipath routing protocol which provides a proactive-like routing with less end-to-end delay and less control overhead. Also a fast error recovery scheme to cope with the potential route failures caused by node destruction by the enemy is proposed. The drawback of this approach is that load balancing and more efficient route maintenance is not taken into account.

Khazaei and Berangi (2009) have proposed a multipath routing with fault tolerance technique in MANETs. Their protocol is an extension of DSR for enhancing the reliability by modifying the route discovery and route maintenance processes in DSR. The multiple routes are maximally node-disjoint in this protocol. Their approach does not consider the quality of service into account for data transmission.

\section{MATERIALS AND METHODS}

\section{Multipath routing protocol for effective local route recovery:}

Overview: We proposed a hybrid routing protocol involving multipath discovery and route recovery. When the source node needs to forward a data packet to a destination, it floods the Route Request (RREQ) packet to the entire network. The intermediate node updates its route caches about the routing information whenever it receives the RREQ packet and continues packet broadcasting. The destination node upon receiving all RREQ packets attaches the route code constituting available bandwidth information and feedback Route Reply (RREP) packets. Upon reception of RREP packets, the source node selects the primary route based on the route code. In case of route failure in the primary route, the recovery node detects it and establishes a local recovery path with maximum bandwidth from its route cache. The route recovery management technique is handled to avoid the frequent collision and degradation in the network performance.

Available bandwidth estimation: After RREQ packets are forwarded, time slots of every route between source and destination is collected by the destination node at the same time. Thus the destination node maintains the status for time slots related to every route.

With the help of time slots in the destination node, the route of maximum bandwidth can be found using following computation.

We assume that $\mathrm{N}$ routes $\left(\mathrm{n} 1, \mathrm{n} 2, \ldots, \mathrm{n}_{\mathrm{N}}\right)$ are discovered between source and destination nodes.

Using the function "Set_Tentative", bandwidth B of route $\mathrm{n}_{\mathrm{i}}(\mathrm{i}=1, \ldots, \mathrm{N})$ can be computed as follows:

$\mathrm{B}_{\mathrm{i}}=\operatorname{Min}\left(\mathrm{T} / 2, \mathrm{~T}_{\mathrm{bi}}\right)$

Where:
$\mathrm{T}=$ Number of total time slots in every route

$\mathrm{T}_{\mathrm{bi}}=$ Number of free time slots in the bottleneck link of route $\mathrm{N}_{\mathrm{i}}$

Multipath routing: The following steps are involved in the multipath routing technique

Step1: Whenever a data packet needs to forwarded by the source node to the destination and no route is found in the cache, then the source floods the route request (RREQ) packets to the entire network.

Step 2: If the non-duplicate RREQ packet reaches the intermediate nodes, it attaches the node ID to packet and continues broadcasting.

Step 3: If duplicate packets are received, instead of dropping all duplicate packets, only those that are received from the longer routes are dropped thus minimizing the packet drop.

Step 4: Even if, route information to the destination is present in the route cache of intermediate node, it has no permission to send a Route Reply (RREP) back to the source and permission is given only to the destination node.

Step 5: The destination node upon receiving all RREQ packets attaches the route code and feeds it back as RREP packets. Let $n$ RREP packets are generated for the paths $\mathrm{P}_{1}, \mathrm{P}_{2}, \ldots, \mathrm{P}_{\mathrm{n}}$. The route code is to recognize the available bandwidth. The RREP with route code $\mathrm{R}_{\mathrm{Cl}}$ has a maximum available bandwidth and RREP with route code $\mathrm{R}_{\mathrm{C} 2}$ has next maximum bandwidth availability and so on. The priority condition for bandwidth selection is as follows:

$$
\mathrm{B}_{1}>\mathrm{B}_{2}>\mathrm{B}_{3}>\mathrm{B}_{4} \ldots>\mathrm{B}_{\mathrm{n}}
$$

where $B_{1}, B_{2}, B_{3}, \ldots, B_{n}$ are the available bandwidth of the routes

Step 6: After the intermediate node receives RREP packets, they store the routes $\mathrm{P}_{1}, \mathrm{P}_{2}, \ldots, \mathrm{P}_{\mathrm{n}}$ in their route caches and then forward them to subsequent nodes.

Step 7: Upon reception of RREP packets, the source node selects the primary route based on the route code.

Step 8: In case of route failure in the primary route, the recovery node detects it and establishes a local recovery path with maximum bandwidth (which is the first available path) from its route cache. The route recovery technique is described as follows. 
Route recovery technique: During the data transmission, the node mobility and low battery power are the issues causing route breakage. To handle this, a local recovery mechanism is triggered which is based on the establishment of recovery nodes.

The sequence of steps involved in the route recovery technique is as follows:

- The nodes that overhear transmitted data on the $\mathrm{k}$ sequence of nodes in the primary nodes are selected as recovery nodes

- In case the primary route attempts transmission of the data packet to the failed route, the recovery node detects it and initiates route recovery phase

- The recovery nodes listen to the retransmission and then waits for overhear acknowledgement. $\mathrm{O}_{\text {Ack }}$

- If no $\mathrm{O}_{\text {Ack }}$ is heard, then the recovery nodes forwards Vary Route (VR) packet to the node that attempts retransmission

- When the node receives VR, it updates the route cache and forwards the Ack to recovery node

- When the recovery node receives the Ack, it chooses the first route in its route cache since it has the maximum bandwidth and use that recovery route to retransmit the data packet. In case the first route is busy or cannot be established, it fetches the next route from the cache and so on.

Route recovery management: The network may possess many recovery nodes in the dense environment. In case of the route failure, all the existing recovery nodes attempts route recovery, by sending VR simultaneously. This results in frequent collisions and degradation of the network performance.

To overcome this drawback, we consider recovery route management technique which is as follows.

Every node has a various contention window (C) dimensions as per the overhearing count's number. If the number is large, the nodes $\mathrm{C}$ dimension is small. This reveals that the recovery node related to the primary route is more stable than other routes. The recovery node selects $\mathrm{C}$ in a random manner and waits for time $t$. In case recovery node hears VR message sends by another recovery node, the timer is stopped. If VR is not sent by any node within the time interval $t$, then the recovery node forwards VR for the purpose of route discovery.

In particular, our route management scheme restricts the collision avoidance to be performed by the first VR message. There may be probability that first VR may collide with VR of other recovery nodes having the similar $\mathrm{C}$ value and it is defined as:

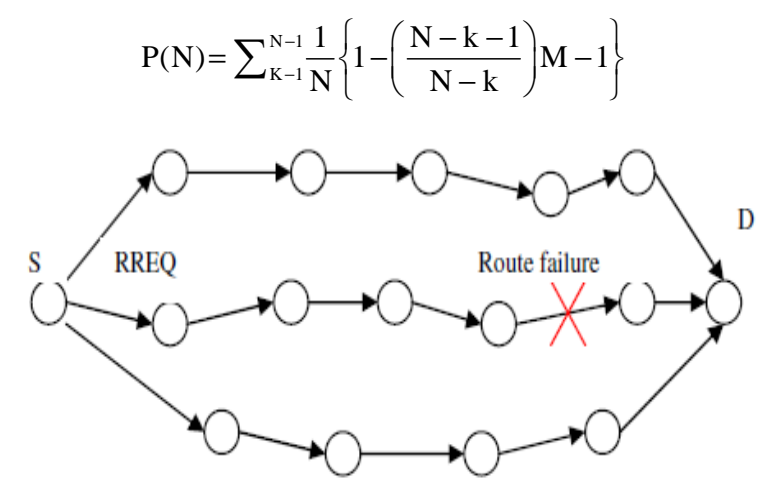

Fig. 1: Route failure

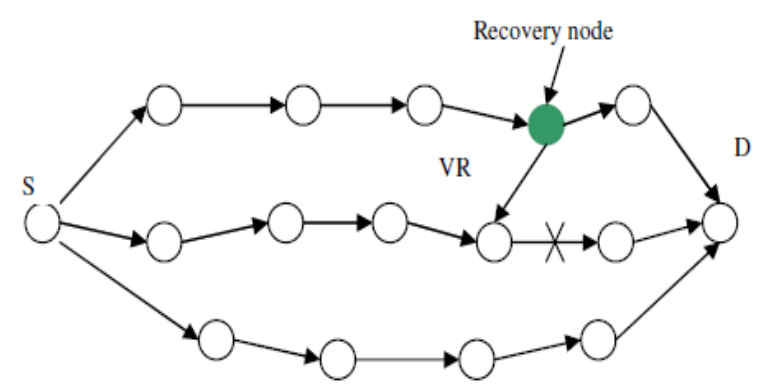

Fig. 2: Recover node establishment and forwarding "Vary Route" (VR) message to node attempting retransmission

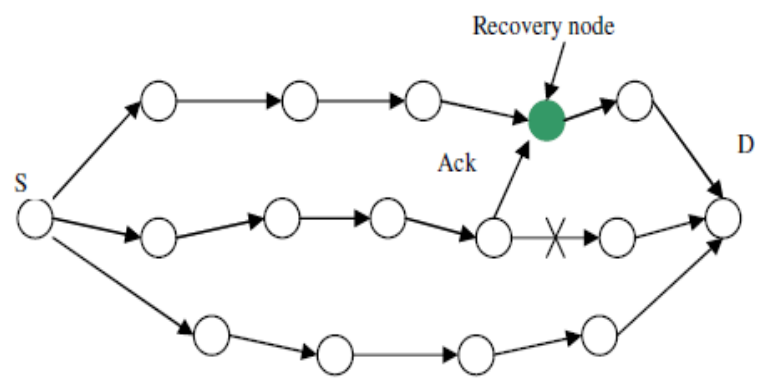

Fig. 3: Acknowledging recovery node

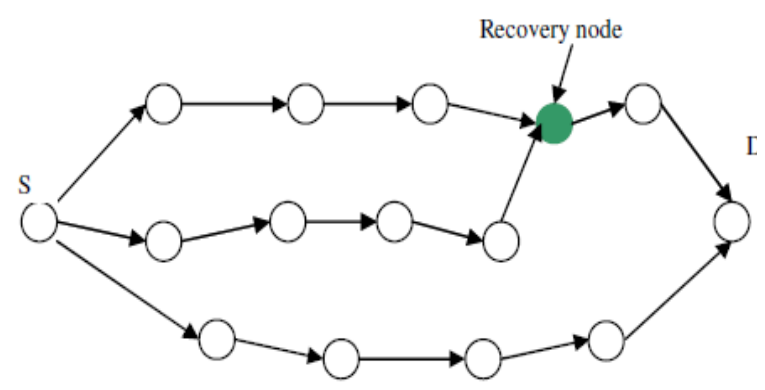

Fig. 4: New route recovery 
where, $\mathrm{M}$ is the number of recovery nodes and the range of $\mathrm{C}$ size is from 0 to $\mathrm{N}$.

The mobility of recovery node causes it to misunderstand that route as failed even though original route is available to transmit the data. If the recovery node forwards VR message to a node which connects to the subsequent node well, it discards the VR and further recovery node is conscious about its misjudgment because it does not receive the Ack.

The steps involved in the route recovery technique are depicted in the following Fig. 1-4.

\section{RESULTS AND DISCUSSION}

Simulation model and parameters: We use NS2 to simulate our proposed protocol. In our simulation, the channel capacity of mobile hosts is set to the same value: 2 Mbps. We use the Distributed Coordination Function (DCF) of IEEE 802.11 for wireless LANs as the MAC layer protocol. It has the functionality to notify the network layer about link breakage.

In our simulation, mobile nodes of sizes 25, 50, 75, 100 and 125 move in a $1000 \times 1000 \mathrm{~m}$ region for 20 seconds simulation time. We assume each node moves independently with the same average speed. All nodes have the same transmission range of 250 $\mathrm{m}$. In our simulation, the minimal speed is $5 \mathrm{~m} \mathrm{sec}^{-1}$ and maximal speed is $25 \mathrm{~m} \mathrm{sec}^{-1}$. The speed is varied as 5, 10, 15, 20 and $25 \mathrm{sec}$. The simulated traffic is Constant Bit Rate (CBR).

Our simulation settings and parameters are summarized in Table 1.

Table 1: Simulation parameters

\begin{tabular}{ll}
\hline No. of nodes & $25,50,75,100$ and 125 \\
\hline Area size & $1000 \times 1000$ \\
Mac & 802.11 \\
Radio range & $250 \mathrm{~m}$ \\
Simulation time & $20 \mathrm{sec}$ \\
Traffic source & $\mathrm{CBR}$ \\
Packet size & 512 \\
Mobility model & Random way point \\
Rate & $100 \mathrm{~Kb}$ \\
Max. packet in queue & 150 \\
Speed & $5,10,15,20$ and 25 \\
\hline
\end{tabular}

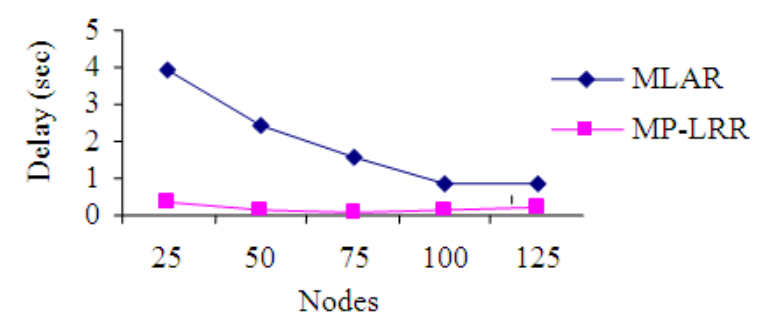

Fig. 5: Nodes Vs delay
Performance metrics: We compare our MP-LRR protocol with the MLAR (Trung et al., 2007) protocol. We evaluate mainly the performance according to the following metrics.

Average end-to-end delay: The end-to-end-delay is averaged over all surviving data packets from the sources to the destinations.

Average packet delivery ratio: It is the ratio of the number of packets received successfully to the total number of packets sent.

Drop: It is the number of packets dropped during the data transmission.

Control overhead: The control overhead is defined as the total number of routing control packets normalized by the total number of receiving data packets.

Based on nodes: In the initial experiment we vary the number of Nodes as 25, 50, 75,100 and 125.

From Fig. 5, we can see that the average end-to-end delay of the proposed MP-LRR protocol is less when compared to the MLAR protocol.

From Fig. 6, we can see that the packet delivery ratio for MP-LRR increases, when compared to MLAR, since it utilizes robust links.

From Fig. 7, we can see that the packet drop for MP-LRR is less, when compared to MLAR.

Figure 8 shows the control overhead of the protocols. The values are considerably less in MP-LRR when compared with MLAR.

Based on speed: In the second experiment we vary the Speed value as 5, 10, 15, 20 and 25 .

From Fig. 9, we can see that the average end-to-end delay of the proposed MP-LRR protocol is less when compared to the MLAR protocol.

From Fig. 10, we can see that the packet delivery ratio for MP-LRR increases, when compared to MLAR, since it utilizes robust links.

From Fig. 11, we can see that the packet drop for MP-LRR is less, when compared to MLAR.

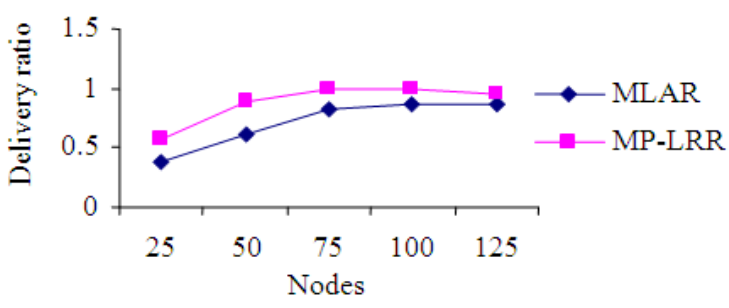

Fig. 6: Nodes Vs delivery ratio 
J. Computer Sci., 8 (7): 1143-1149, 2012

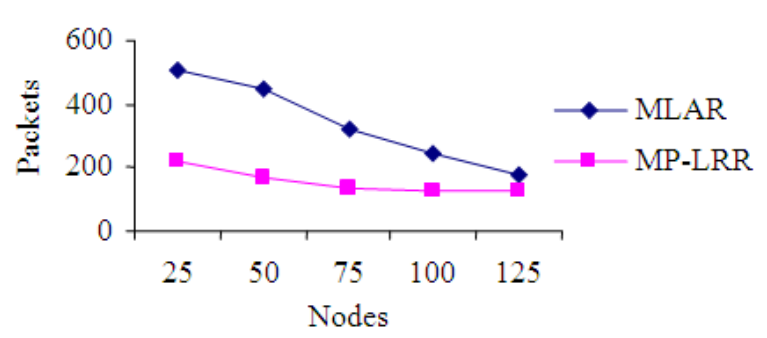

Fig. 7: Nodes Vs drop

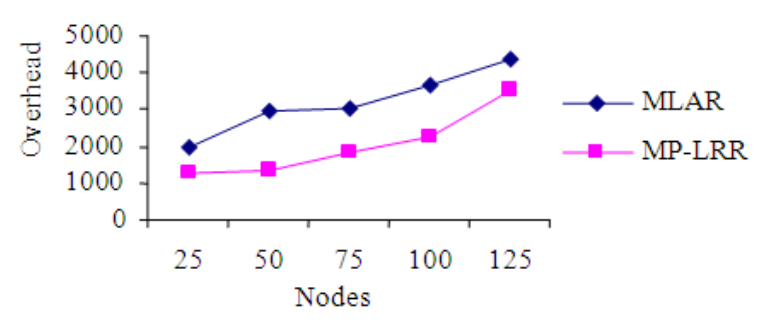

Fig. 8: Nodes Vs overhead

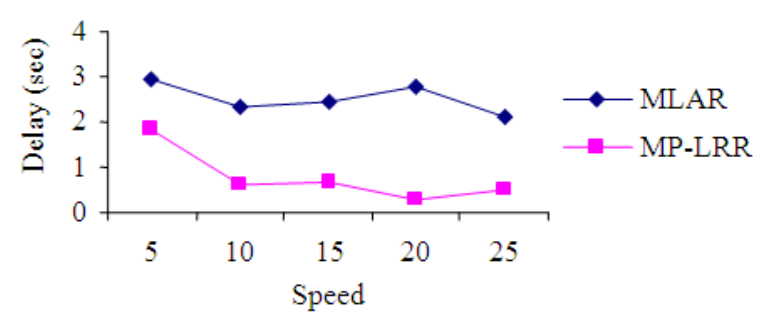

Fig. 9: Speed Vs delay

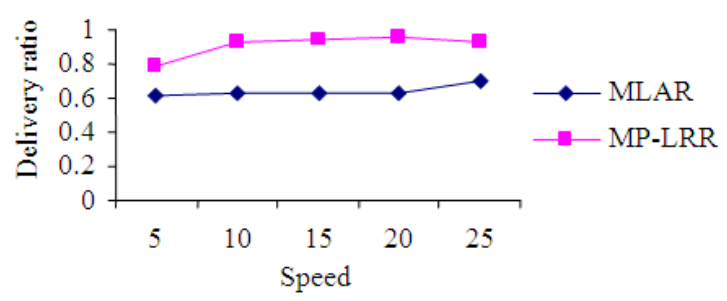

Fig.10: Speed Vs delivery ratio

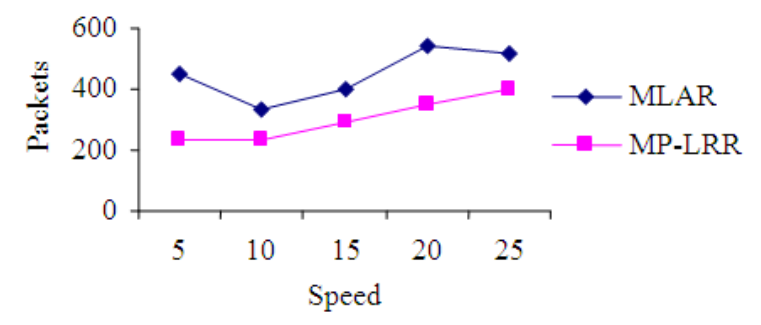

Fig.11: Speed Vs drop

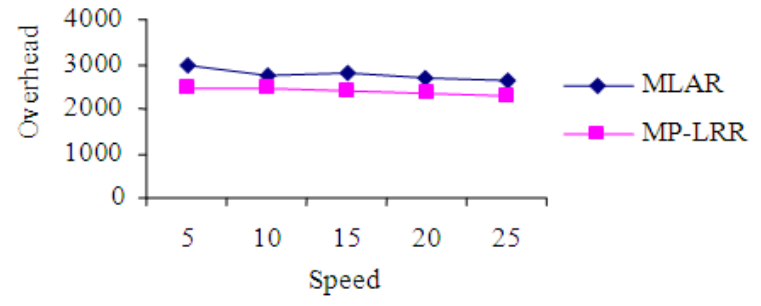

Fig. 12: Speed Vs overhead

Figure 12 shows the control overhead of the protocols. The values are considerably less in MP-LRR when compared with MLAR.

\section{CONCLUSION}

In this, we propose multipath routing protocol for effective local route recovery in Mobile Ad Hoc Network (MANET). When the source requires forwarding a data packet to a destination, it floods the Route Request (RREQ) packet to the entire network. The intermediate node on receiving RREQ packet updates its route caches and rebroadcast the packet. The destination node upon receiving all RREQ packets attaches the route code constituting available bandwidth information and feedback RREP packets.

The intermediate node updates the routing information in its route cache on receiving the RREP packet. When RREP reaches the source node, it selects primary route based on the route code. In case of route failure in the primary route, the recovery node detects it and establishes a local recovery path with maximum bandwidth from its route cache. The route recovery management technique is handled to avoid the frequent collision and degradation in the network performance. By simulation results, we have shown that the proposed approach improves network performance.

\section{REFERENCES}

Aminu, M., M. Ould-Khaoua, L.M. Mackenzie, C. Perkins and J.D. Abdulai, 2009. Probabilistic counter-based route discovery for mobile ad hoc networks. Proceedings of the 2009 International Conference on Wireless Communications and Mobile Computing: Connecting the World Wirelessly, (WCMC' 09) ACM New York, NY, USA, $\quad$ pp: 1335-1339. DOI: $10.1145 / 1582379.1582672$

Dhirendra, K.S., S. Kumar and C. Kumar, 2010. Enhancement of split multipath routing protocol in MANET. Int. J. Comput. Sci. Eng., 02: 679-685. 
Juanwei, L., J. Chen and Y. Kuo, 2009. Multipath routing protocol for network lifetime maximization in ad-hoc networks. Proceedings of the 5th International Conference on Wireless Communications, Networking and Mobile Computing, (WCNMC' 09), IEEE Xplore Press, Piscataway, NJ, USA, pp: 2713-2716. DOI: 10.1109/WICOM.2009.5305828In

Kang, B.S. and I.Y. Ko, 2010. Effective route maintenance and restoration schemes in mobile ad hoc networks. Sensors, 10: 808-821.

Keming, D.U. and Y. Yang, 2010. A QoS routing for maximum bandwidth in ad hoc networks. Proceedings of the 2nd International Conference on Future Networks (ICFN), Jan. 22-24, Sanya, Hainan, pp: 343-345. DOI: 10.1109/ICFN.2010.11

Khazaei, M. and R. Berangi, 2009. A multi-path routing protocol with fault tolerance in mobile ad hoc networks. Proceedings of the 14th International CSI Computer Conference, Oct. 20-21, IEEE Xplore Press, Tehran, pp: 77-82. DOI: 10.1109/CSICC.2009.5349359

Khetwal, N. and Sharma, 2012. Multimedia based dynamic source routing for mobile ad hoc networks. J. Inform. Operations Manage., 3: 213216.
Seethalakshmi, P., M.J.A. Jude and G. Rajendran, 2011. An optimal path management strategy in a mobile ad hoc network using fuzzy and rough set theory. Am. J. Applied Sci., 1314-1321. DOI: 10.3844/ajassp. 2011.1314.1321

Suganthi, P. and A. Tamilarasi, 2012. Impact of malicious nodes under different route refresh intervals in ad hoc network. Am. J. Applied Sci., 18-23. DOI: 10.3844/ajassp. 2012.18.23

Sung-Won, L., J.Y. Choi, K.W. Lim, Y. Ko and B. Roh, 2010. A reliable and hybrid multi-path routing protocol for multi-interface tactical ad hoc networks. Proceedings of the Conference on Military Communication Unclassified Program Networking Protocols and Performance Track, (MCUPNPP' 10), pp: 1531-1536.

Trung, H.D., W. Benjapolakul and P.M. Duc, 2007. Performance evaluation and comparison of different ad hoc routing protocols. Comput. Commun., $\quad 30$ : 2478-2496. DOI: 10.1016/j.comcom.2007.04.007

$\mathrm{Wu}$, Y.C. and C.C. Tuan, 2009. Power saving routing protocol with power sieving in wireless ad hoc networks. Proceeding of the International Conference on Networks Security, Wireless Communications and Trusted Computing, April 25-26, IEEE Xplore Press, Wuhan, Hubei, pp: 349352. DOI: $10.1109 /$ NSWCT.2009.36 